\title{
TNF Produced by Inflammatory Blood Mononuclear Cells Directly Contributes to Cartilage Damage in Arthritis
}

Anna Yarilina ( $\square$ anna.yarilina@abbvie.com )

Abbvie Bioresearch Center https://orcid.org/0000-0002-7925-856X

Terry Melim

Abbvie Bioresearch Center

Zehra Kaymakcalan

Abbott Bioresearch Center

Research article

Keywords: TNF, D2E7, chondrocytes, cartilage, fibroblast-like synoviocytes, rheumatoid 37 arthritis, blood mononuclear cells

Posted Date: January 8th, 2020

DOI: https://doi.org/10.21203/rs.2.20348/v1

License: (c) (i) This work is licensed under a Creative Commons Attribution 4.0 International License. Read Full License 


\section{TNF Produced by Inflammatory Blood Mononuclear \\ 2 Cells Directly Contributes to Cartilage Damage in \\ 3 Arthritis}

4

5

6
Anna Yarilina, Terry Melim, Zehra Kaymakcalan

Global Biologics Department and Pharmacology Department, AbbVie Bioresearch Center, 100 Research Drive, Worcester, MA, 01605, United States

Corresponding author: Anna Yarilina 


\section{Abstract (<350)}

2 Background: Anti-TNF therapies are effective at preventing inflammation and structural damage in rheumatoid arthritis (RA). However, the role of TNF in cartilage destruction in RA is not well understood. Therefore, we studied the effects of TNF on cartilage and compared TNF production by different cell types involved in joint pathology.

Methods: Primary human chondrocytes and cartilage explants were cultured with recombinant TNF. Bovine cartilage was co-cultured with activated human peripheral blood mononuclear cells (PBMCs) or fibroblast-like synoviocytes (FLS).

10 Expression of cytokines and metalloproteinases (MMPs) was assessed by qPCR 11 and MSD, and proteoglycan depletion from cartilage was assessed using 12 histomorphometry and colorimetric detection in tissue culture supernatants. D2E7 was 13 used to block TNF both in vitro and in vivo in a human TNF transgenic (hTNF-Tg) 14 mouse model of arthritis.

15 Results: TNF elicited strong pro-inflammatory and catabolic effects on isolated human chondrocytes and cartilage explants leading to upregulation of IL-6 and MMPs, as well as proteoglycan depletion from bovine cartilage explants. In an effort to identify cellular sources of TNF, we challenged chondrocytes, FLS and PBMCs with inflammatory stimuli present in RA joints and found that PBMC that were used to model inflammatory cell infiltration produced significantly higher levels of TNF. Moreover, co-culture with activated PBMCs resulted in proteoglycan depletion from bovine cartilage explants. In sharp contrast with stromal cells, TNF failed to induce high amounts of IL-6 and MMPs in PBMCs, suggesting that different cell populations play distinct roles in the triggering and propagation of joint destruction. TNF blockade protected cartilage from damage both in co-culture systems and in a hTNF-Tg mouse model of arthritis.

Conclusions: Our data demonstrate that TNF directly triggers a catabolic program in human chondrocytes leading to cartilage damage and further suggest that neutralization of TNF produced by immune cells infiltrating the inflamed joints, decreases catabolic activity of chondrocytes and fibroblasts, which, in turn, contributes to the cartilage protective effects of anti-TNF biologics in arthritis.

\section{Keywords}

37 TNF, D2E7, chondrocytes, cartilage, fibroblast-like synoviocytes, rheumatoid

38 arthritis, blood mononuclear cells 


\section{Background}

2 Rheumatoid arthritis (RA) is a chronic autoimmune inflammatory disease of unknown

3 aetiology that preferentially targets peripheral joints. RA pathology is highly

4 heterogeneous and involves activation of both adaptive and innate immune responses,

5 dysregulated cytokine expression and hyperactivation of stromal cells that support

6 disease progression (1-3). The use of targeted therapies in clinical studies of RA have

7 elucidated the role of different mechanisms in disease pathogenesis and demonstrated

8 that pathways activated by cytokines, such as tumor necrosis factor (TNF) and

9 interleukin 6 (IL-6), are crucial for RA $(1,3,4)$.

10 Articular cartilage is one of the target tissues in RA. It covers the surfaces of long

11 bones forming the joint and consists of avascular highly organized dense extra-

12 cellular matrix (ECM) populated by chondrocytes. Cartilage ECM is synthetized by

13 chondrocytes and is composed of a collagen network (predominantly type II collagen)

14 and the proteoglycan (PG) aggrecan (5). Several mechanisms contributing to cartilage

15 damage in RA have been proposed. TNF and other pro-inflammatory cytokines found

16 in synovial fluid have been shown to directly damage cartilage by inducing apoptosis

17 of chondrocytes or by increasing production by chondrocytes of proteolytic enzymes,

18 such as metalloproteinases (MMPs) and aggrecanases, even before the development

19 of synovial inflammation (5-9). Cartilage destruction is also associated with synovial

20 inflammation and directly mediated by invasion of hyperplastic inflamed synovial

21 tissue called pannus. Pannus is a highly aggressive tumor-like tissue that mainly

22 consists of activated fibroblast-like synoviocytes (FLS) with infiltrates of immune

23 cells migrating from peripheral blood to inflamed synovium $(3,7,10)$. Activated FLS

24 show elevated expression of cytokines, chemokines, adhesion molecules and

25 proteolytic enzymes that propagate an aggressive invasion of the pannus with

TNF directly contributes to cartilage damage in arthritis 
1 destruction of cartilage matrix $(5,11-13)$. RA FLS have been shown to maintain a

2 unique "transformed" phenotype and retain the ability to invade and damage cartilage

3 both ex-vivo when co-cultured with cartilage explants and in vivo when injected in

4 mice $(11,14-16)$.

5 Clinical studies have demonstrated that anti-TNF therapy inhibits both inflammation

6 and structural damage of bone and cartilage. However, the role of TNF in triggering

7 and propagating cartilage damage in RA is not clear. Results from in vivo studies in

8 knockout mice suggest that cartilage destruction is indirectly regulated by TNF via an

9 increase in IL-1 $\beta$ production by hematopoietic cells (17). Other reports suggest that

10 TNF present in synovial fluid directly induces apoptosis of chondrocytes or mediates

11 cartilage damage by inducing production of proteases by chondrocytes $(6-9,18,19)$.

12 Recent therapies with synthetic disease-modifying antirheumatic drugs (DMARDs)

13 and biologics have significantly improved clinical outcomes of RA patients and have

14 even induced clinical remission in some cases (20-23). However, even the most

15 efficacious RA treatments that successfully inhibit inflammation and progression of

16 joint destruction are incapable of reversing the structural damage of bone and

17 cartilage and fully restoring joint function $(24,25)$. In addition, results of clinical and

18 animal studies suggest that synovial inflammation and tissue destruction may be

19 uncoupled and that structural changes in cartilage and bone may occur and progress in

20 the absence of clinical markers of active inflammation $(12,17,26,27)$. Conversely,

21 therapies directed against bone damage helped reduce the radiological damage but

22 had no effect on inflammation (28). Recent elucidation of the relationship between

23 structural damage and functional outcomes have led to dramatic changes in the

24 management of RA. The new strategic approach, treat-to-target, aims to minimize

25 structural damage and achieve the optimal outcome by early aggressive disease

TNF directly contributes to cartilage damage in arthritis 
1 intervention, stringent criteria of remission and tight control of disease activity (3, 29-

$232)$.

3 In the present study, we addressed the direct role of TNF in RA cartilage damage and

4 compared TNF production by different cell types involved in joint pathology in RA to

5 better understand the mechanism of cartilage protection by TNF blocking

6 therapeutics.

\section{Materials and methods}

\section{Cells}

9 Primary human FLS, chondrocytes and human cartilage explants were purchased

10 from Articular Engineering. Human peripheral blood mononuclear cells (PBMCs)

11 were isolated by density gradient centrifugation from the whole blood of healthy

12 donors purchased from BioIVT.

\section{Reagents}

14 The following reagents were used as indicated: $10 \mathrm{ng} / \mathrm{ml}$ of recombinant human TNF

15 and IL-1 $\beta$ (R\&D Systems), $1 \mathrm{ng} / \mathrm{ml}$ of lipopolysaccharide (LPS, E. coli 0127:B8;

$16 \mathrm{Sigma}$ ), $10 \mu \mathrm{g} / \mathrm{ml}$ of recombinant human S100A8 and S1009 proteins (AbbVie), 5

$17 \mu \mathrm{g} / \mathrm{ml}$ of anti-human TNF antibody D2E7 (human IgG1, AbbVie) and control human $18 \operatorname{IgG1}$ (Sigma).

\section{Cell culture}

20 FLS and chondrocytes were first expanded in T75 flasks in Dulbecco minimum

21 essential medium (DMEM; Invitrogen) or DMEM/F12 (Invitrogen) respectively,

22 supplemented with $10 \%$ heat inactivated defined fetal bovine serum (FBS; HyClone),

23 GlutaMAX Supplement, 0.02M HEPES and Pen/Strep (100 units/mL of penicillin and TNF directly contributes to cartilage damage in arthritis 
$1 \quad 100 \mu \mathrm{g} / \mathrm{mL}$ of streptomycin; all from Invitrogen), and then were used for experiments

2 between passages 2 and 3. FLS and PBMC were seeded on 12-well plates at $10^{5}$

3 cells/well and $1.5 \times 10^{6}$ cells/well, respectively, and cultured in $1.5 \mathrm{ml}$ of DMEM

4 supplemented with 10\% FBS, GlutaMAX, 0.02M HEPES and Pen/Strep.

5 Chondrocytes were cultured in 12-well plates at $10^{5}$ cells/well in Advanced

6 DMEM/F12 (Invitrogen) supplemented with 0.02M HEPES, GlutaMAX and

7 Pen/Strep. Following 24 h equilibration, cells were treated with different stimuli and

8 anti-TNF antibody were used in some of the experiments in concentrations indicated

9 above.

In vitro cartilage destruction assay

11 Bovine cartilage explants (3 $\mathrm{mm}$ in diameter) were produced from one

12 metacarpophalangeal joint of a skeletally mature animal (Articular Engineering).

13 Explants were placed in 96-well flat bottom plates (1 explant/well) and human FLS

$14\left(5 \times 10^{3}\right.$ cells/well $)$ or PBMCs $\left(2 \times 10^{5}\right.$ cells/well $)$ were seeded over the cartilage pieces

15 in $200 \mu \mathrm{l}$ of complete Advanced DMEM/F12 or DMEM/F12 media respectively.

16 Cartilage mono- and co-cultures were treated with the same stimuli and $\mathrm{Ab}$ as

17 described above for 14 days (4 explants per treatment condition). Media was

18 exchanged every 3 days and saved for future analyses. At the end of culture, cartilage

19 pieces were collected for PG analysis.

\section{Cytokine, MMP and proteoglycan detection}

21 Culture media were collected at various times and levels of cytokines and MMPs

22 were detected using mesoscale discovery (MSD) assays. PG depleted from cartilage

23 into the medium was measured using a sGAG Assay Kit (Kamiya Biomedical Co)

24 following the manufacturer's protocol.

TNF directly contributes to cartilage damage in arthritis 


\section{Arthritis model}

2 In vitro effects of D2E7 on cartilage were evaluated in the hTNF-Tg mouse model of

3 arthritis. hTNF-Tg mice (Tg197 strain, C57BL/6 genetic background) spontaneously

4 develop inflammatory, destructive arthritis upon constitutive hTNF overexpression.

5 Both the genotype and phenotype of these mice have been described previously in more

6 detail (33). Briefly, the animals develop joint swelling in the small joints of the front

7 and hind paws between weeks 5 and 6 after birth. This chronic polyarthritis progresses

8 over time and results in severe joint damage. In this study, $\operatorname{Tg} 197$ mice heterozygous

9 for human TNF were injected intraperitoneally (i.p.) with $1 \mathrm{mg} / \mathrm{kg}$ of anti-TNF $\alpha$ 10 antibody D2E7 $(n=12)$ or placebo $(n=6)$ once a week for 12 weeks. At the end of the 11 study hind paws were collected and processed for histochemistry. Animals were housed 12 and maintained at the facilities of the Hellenic Pasteur Institute, under specific pathogen

13 free (SPF) conditions. All experimental protocols were reviewed and approved by the 14 Institutional Committee for Animal Care of Hellenic Pasteur Institute, and by Abbott's 15 Corporate Animal Welfare Committee.

\section{Tissue processing and staining}

17 In vitro studies. At the end of culture, bovine cartilage explants were washed in 18 phosphate buffered saline (PBS; Invitrogen), fixed in 10\% neutral buffered formalin 19 (Alfa Aesar) for 24 hours at room temperature, cut in half, embedded in optimal cut temperature compound (OCT; Tissue-Tek) and kept frozen at $-80^{\circ} \mathrm{C}$. Frozen cartilage

21 tissues were sectioned (5 $\mu \mathrm{m}$ thick) using a tape transfer technique and were stained 22 with toluidine blue for PG detection. Slides were scanned on the Vectra Imaging 23 system at low power. PG content (as measured by the toluidine blue optical density 24 (OD)) was analysed histomorphometrically as follows: high power (20x) images were selected on each cartilage sample (minimum 2 images per cartilage piece) and TNF directly contributes to cartilage damage in arthritis 
1 analysed by InForm (Perkin Elmer) image analysis software. The data represent an

2 average of OD of the dye (the amount of absorbing material present at the area of

3 interest) in the image of cartilage pieces that underwent the same treatment (one OCT

4 block).

5 In vivo studies in hTNF-Tg mice. Hind legs were fixed in 10\% neutral buffered

6 formalin, decalcified, embedded in paraffin, and $5 \mu \mathrm{m}$ ankle joint sections were

7 prepared and stained with hematoxylin (detects nuclei) and alcian blue (PG detection).

8 Total cartilage area and area stained with alcian blue on the proximal and distal ends

9 of talus bones were measured, and PG content was determined as a percent of alcian

10 blue-positive areas within total cartilage area.

\section{Statistical analysis}

12 Statistical analysis was performed using GraphPad Prism analytical software version

138 for Windows. Statistical tests included the nonparametric Mann-Whitney U test,

14 Kruskal-Wallis test with Dunn's post-test for multiple comparisons; repeated measure

15 two-way analysis of variance (ANOVA) with Sidak's post hoc tests for multiple

16 comparisons, two- and one-way ANOVA with Tukey's or Dunn's post hoc tests for

17 multiple comparisons. $P$ values less than 0.05 were considered significant.

\section{Results}

19 TNF induces sustained expression of pro-inflammatory cytokines and MMPs in

20 human chondrocytes and triggers cartilage destruction

21 Our studies focused on TNF-mediated activation of human chondrocytes and their

22 production of pro-inflammatory cytokines and MMPs that degrade type II collagen

23 and PGs, two major components of cartilage $(34,35)$. First, we compared

24 inflammatory responses of isolated chondrocytes with that of chondrocytes that TNF directly contributes to cartilage damage in arthritis 
1 remain embedded in cartilage tissue. We cultured cartilage explants or cells isolated

2 and grown as a monolayer in the presence or absence of TNF for several days and

3 evaluated expression of mediators of inflammation and tissue damage. Upon TNF

4 treatment, chondrocytes secreted high levels of IL-6, MMP1 and MMP3 that were

5 sustained and increased over time, reaching maximum levels ( $\mathrm{ng} / \mathrm{ml}$ for IL-6 and

$6 \mu \mathrm{g} / \mathrm{ml}$ for MMPs) between 24 and 72 hours after TNF addition to cultures (Fig. 1A

7 and data not shown). The analysis of IL-6, MMP1 and MMP3 mRNA revealed a

8 similar pattern of continuous expression in response to TNF (data not shown). We

9 obtained comparable results with cultured cartilage explants and isolated

10 chondrocytes (Fig. 1A, upper and lower panels), supporting previous findings that

11 isolated cells at early passages (P 2-3) maintain characteristics typical of chondrocytes

12 within the cartilage and thus are suitable for in vitro studies of chondrocyte biology

$13(16,35)$.

14 Next, we examined whether increased production of MMPs by TNF-activated

15 chondrocytes translated into cartilage damage. For in vitro cartilage destruction

16 studies, we used bovine cartilage as a well-established and readily available

17 experimental model that is commonly used for studies of human cytokines and growth

18 factors (16). Bovine cartilage explants were cultured in the presence or absence of

19 TNF for 14 days. IL-1 $\beta$, known to be a strong cartilage catabolic factor, served as a

20 positive control. Media with cytokines were replenished every 3 days. At the end of

21 experiments, cartilage pieces were collected, embedded in OCT, sectioned and PG

22 content was detected by toluidine blue staining. TNF treatment resulted in partial

23 depletion of PG from cartilage, as depicted by partial loss of blue colour (Fig. 1B,

24 upper panel), whereas IL-1 $\beta$-treated tissue was almost completely depleted of PG and

25 did not retain the dye (Fig. 1B, upper panel). Image analysis results from multiple

TNF directly contributes to cartilage damage in arthritis 
1 experiments confirmed that although TNF was less potent than IL-1 $\beta$, it caused

2 significant depletion of PG from the cartilage as compared to untreated controls (Fig.

$31 \mathrm{~B}$, lower panel). We also found that IL-1 $\beta$ induced higher levels of IL- 6 and MMPs

4 compare to TNF both in isolated chondrocytes and cartilage cultures (Fig.1C). Our

5 results suggest that TNF induces a sustained inflammatory and tissue destructive

6 program in chondrocytes that leads to matrix degradation and destruction of cartilage

7 in vitro.

8 Co-culture with activated FLS has a marginal effect on proteoglycan depletion

9 from the cartilage

10 To gain a better understanding of the mechanisms underlying cartilage protection by 11 anti-TNF biologics, we established an experimental system whereby bovine cartilage 12 was co-cultured with activated human cells relevant to RA pathology. RA FLS have 13 been reported to invade articular cartilage in vitro and in vivo $(16,36)$ and secrete a 14 wide array of factors that mediate activation and survival of synovial inflammatory 15 cells, enhance synovial neo-angiogenesis, and induce cartilage degradation $(11,37)$. 16 To evaluate catabolic effects of activated FLS, bovine cartilage explants were co17 cultured with human FLS isolated from the joints of RA patients, or from individuals 18 without joint pathology, in the presence or absence of recombinant human TNF, and 19 PG depletion from the explants was measured using toluidine blue. Surprisingly, we 20 found that neither untreated nor TNF-activated RA FLS increased PG depletion, 21 whereas non-diseased FLS treated with TNF marginally increased explant destruction 22 as compared to cartilage monoculture (Figure 2A). We further compared expression of 23 matrix degrading enzymes in activated FLS and found that after stimulation with 24 TNF, RA FLS produced less MMP1 and MMP3 than non-diseased cells (Fig. 2B). This 
1 finding is in-line with the weak catabolic effect of TNF-treated RA FLS on cartilage

2 observed in Figure 2A.

3 Next, we tested if neutralization of endogenous TNF protects cartilage from damage.

4 To initiate the catabolic program, human FLS/bovine cartilage co-cultures were treated

5 with TNF or IL-1 $\beta$ for three days following the addition of D2E7 monoclonal antibody

6 to block TNF. D2E7 selectively inhibited production of IL-6 and MMPs by FLS in co-

7 culture systems (Fig. 2D and data not shown) and protected cartilage explants from PG

8 depletion in mono- and co-cultures treated with TNF but not with IL-1 $\beta$ (Fig. 2C).

9 Overall, our data demonstrate that co-culture with activated FLS had minimal effect on

10 cartilage destruction and that D2E7 protects cartilage cultures from TNF-dependent

11 damage.

\section{Activated PBMCs produce significantly higher levels of TNF compared to joint} stromal cells

Next, we examined whether activated joint stromal cells represent a significant source of endogenous TNF and found that strong pro-inflammatory factors, such as IL-1 $\beta$ and LPS, induced relatively low TNF expression in chondrocytes and FLS (Fig. 3A).

Given that D2E7 had very little effect on IL-1 $\beta$ mediated cartilage damage, this suggested that TNF was not downstream of IL-1 $\beta$ catabolic effects (Fig. 2C).

Therefore, we investigated additional cellular sources and mediators of TNF in RA joints. In arthritis, activated immune cells migrate to joints from the bloodstream, infiltrate the synovium and propagate inflammation and tissue damage $(3,38)$. Within inflamed tissues, multiple damage-associated molecular patterns (DAMPs) are released from the dead or activated cells that are capable of recruiting immune cells and maintaining the chronic inflammatory state in the joints $(39,40)$. Alarmins are a group of endogenous molecules that belong to the DAMP family and are associated TNF directly contributes to cartilage damage in arthritis 
1 with RA pathogenesis (40). Thus, we compared TNF production by human PBMCs,

2 FLS and chondrocytes after activation with alarmins S100A8 and S100A9. Alarmins

3 and LPS (positive control) induced significantly higher levels of TNF in PBMCs

4 compared to stromal cells (Fig. 3B). IL-1 $\beta$ induction was observed also, even though

5 levels were in the lower picogram range. Furthermore, in co-culture with cartilage

6 explants, treatment of human PBMCs with alarmins resulted in catabolic activity

7 similar to the effect of TNF treatment, as measured by release of PG into the culture

8 media (Fig. 4A). To obtain additional mechanistic insights into the pathogenic effects

9 of TNF in arthritis, we compared responses of three different cell types to TNF and

10 found that, in contrast to FLS and chondrocytes, PBMCs failed to produce sustained

11 high levels of MMP3 and IL-6 (Fig. 3C). Thus, our findings support a model in which

12 activated immune cells are major producers of inflammatory cytokines, such as TNF

13 and IL-1 $\beta$, that activate stromal cells in the joints and trigger a cascade of pathologic

14 events culminating in chronic inflammation and tissue destruction in RA.

15 Neutralization of endogenous TNF attenuates inflammation and protects

16 cartilage from PG depletion in vitro and in vivo

17 To confirm that cartilage destruction can be promoted by endogenously produced TNF

18 and can be blocked by an anti-TNF treatment, we added D2E7 or an isotype control

19 antibody to the activated PBMC/cartilage co-cultures and evaluated PG depletion from

20 the cartilage explants. D2E7 protected cartilage from destruction by blocking both

21 exogenously added TNF and endogenous TNF produced by PBMCs activated with

22 S100A proteins (Fig. 4A and B). Next, we evaluated anti-catabolic effects of D2E7 in

23 vivo in the hTNF-Tg model of arthritis. In this model, mice continuously express human

24 TNF and develop spontaneous arthritis between weeks 5 and 6 after birth (33).

25 Prophylactic administration of D2E7 attenuated inflammation and cartilage destruction TNF directly contributes to cartilage damage in arthritis 
1 as depicted by retention of blue dye (asterisk on Fig. 4C, upper panel). Further analysis

2 of alcian blue staining demonstrated a significantly higher content of PG in cartilage of

3 animals treated with D2E7 (Fig. 4C, lower panel).

4 Collectively, we show that TNF directly triggers a catabolic program in human

5 chondrocytes leading to cartilage damage. Our findings also suggest that neutralization

6 of TNF produced by PBMCs infiltrating inflamed joints decreases catabolic activity of

7 chondrocytes and fibroblasts, which contributes to the cartilage protective effects of

8 anti-TNF biologics in RA.

\section{Discussion}

10 There are several mediators of inflammation that contribute to the pathogenesis and

11 propagation of systemic disease in RA leading to structural damage of joint tissues.

12 Inhibition of major molecular inducers of inflammation, such as TNF, IL-6, IL-1 and

13 chemokines, as well as cellular contributors, such as T cells and B cells, have been

14 shown to be effective in various animal models and in humans (3). As a result, we

15 now have several highly effective drugs in the treatment of RA that inhibit

16 inflammation and reduce structural damage. Anti-TNF biologics are among the

17 effective treatments that have been shown to halt structural damage in articular

18 cartilage and bone. To understand the mechanism of cartilage protection by anti-TNF

19 therapeutics, we studied the effect of TNF on cartilage and chondrocytes, investigated

20 cellular sources of TNF in the joint and explored the outcomes of TNF neutralization

21 on cartilage damage in vitro and in a mouse model of arthritis.

22 It is worth noting that prior to this study, the direct effects of TNF on human

23 chondrocytes and cartilage had not been studied extensively. Most of the published

24 data addressed the role of TNF in animal models of RA or used TNF in combination

TNF directly contributes to cartilage damage in arthritis 
1 with other inflammatory cytokines in vitro $(9,16-18)$. In the present work, we examined responses of isolated chondrocytes and cartilage explants to either exogenously added or endogenously produced TNF. Chondrocytes responded directly to TNF stimulation by secreting IL-6 and the cartilage degrading enzymes, MMP1 and MMP3. Similar results were obtained when isolated human chondrocytes or chondrocytes embedded in the human cartilage matrix of the explants were tested. Interestingly, activation by TNF continued for up to three days, as evidenced by the continuously increasing concentrations of these mediators in the culture supernatants and mRNA levels in chondrocytes. To monitor structural damage, bovine cartilage explants were used as a model, as they are more readily available, have uniform characteristics, an intact surface and are more amendable for studies assessing physical damage. Bovine cartilage explants also have an advantage over human samples typically obtained during autopsy or joint replacement surgery, which have highly variable characteristics. Culturing bovine cartilage explants with TNF for 14 days led to depletion of PGs, a hallmark of cartilage structural damage. In agreement with previous findings, IL- $\beta$ was more efficient in causing cartilage destruction than TNF $(16,18)$. The role of IL- $\beta$ in cartilage and bone destruction in RA is well established, especially in animal models of arthritis $(17,41)$. Although the IL-1R antagonist anakinra has been approved for RA treatment $(42,43)$, the overall antiinflammatory effects of anakinra in RA are moderate and significant improvement is achieved in only about $50 \%$ of patients, when compared to anti-TNF treatment. Nevertheless, anakinra demonstrated a strong protective effect in cartilage and bones $(43-45)$.

As FLS are in close proximity to the cartilage and are activated and transformed into a tumor-like invasive pannus in RA, FLS from RA patients or healthy controls were co- 
1 cultured with cartilage explants with or without TNF addition. Co-culture with FLS

2 did not show marked PG depletion over monocultures of cartilage irrespective of FLS

3 origin or presence of TNF in the cultures. TNF mediated effects were neutralized by

4 the addition of the anti-human TNF antibody, D2E7. When FLS/bovine explant co-

5 cultures were stimulated with TNF, both diseased and healthy FLS responded by

6 producing IL-6. However, RA FLS secreted less of the tissue degrading enzymes,

7 MMP-1 and MMP-3, as compared to non-diseased healthy control cells. Although

8 these results may appear to be counterintuitive, one should consider the chronically

9 activated state of FLS taken from RA patients, which can cause RA FLS to become

10 unresponsive to further stimulation in vitro. Recent studies have suggested that

11 previous exposure to an inflammatory environment can result in long lasting

12 epigenetic memory resulting in altered cellular responses to activation (11). A low

13 response of FLS isolated from the joints of RA patients could also be explained in

14 part by heterogeneity in phenotypes and respective functions of these cells from

15 different layers of the synovium $(46,47)$. For example, it has been shown in animal

16 models that only fibroblasts from superficial layers exhibited destructive phenotype

17 and function (47). A recent extensive report by Mizoguchi et al assessed synovial

18 fibroblasts from RA and control OA patients using single-cell transcriptomics, flow

19 cytometry, in vitro analyses and immunohistochemistry of synovium (48). Three

20 different subpopulations of FLS in RA patients located at distinct anatomical sites and

21 possessing different biological functions were identified. In addition, the authors

22 demonstrated that FLS functional phenotype could not be maintained in vitro because

23 of culture conditions and loss of the microenvironment (48). As we did not have

24 information regarding collection area, stage, duration and treatment of disease of the

TNF directly contributes to cartilage damage in arthritis 
1 FLS samples, it is possible that we did not study the relevant FLS subpopulations

2 most important for structural damage.

3 After demonstrating the catabolic effects of exogenously added TNF on cartilage

4 activation and destruction, we next tested whether endogenously produced TNF

5 elicited the same responses. To determine the cellular sources of TNF, we compared

6 TNF production by PBMCs that were used as a model for immune cells migrating to

7 the inflamed synovium from blood, FLS and chondrocytes activated by the alarmins

8 S100A8 and S100A9, which belong to the DAMP family and are present in inflamed

9 joints $(40,49,50)$. The expression of S100A8 and S100A9 is closely correlated with

10 the intensity of inflammation and macrophage activation in mouse arthritis models

11 (51, 52). In addition, mice deficient for S100A9 have reduced joint swelling and

12 proteoglycan depletion relative to wildtype mice, whereas intra-articular injection of

13 S100A8 into wildtype mice induces synovitis, depletes cartilage proteoglycan levels

14 and upregulates synovial levels of mRNAs encoding S100A8, S100A9, IL-1 $\beta$ and

15 MMPs (51, 53). S100A8 and S100A9 treatment resulted in strong TNF production by

16 PBMCs only, whereas TNF production by chondrocytes and synovial fibroblasts was

17 low. Furthermore, S100A8 or S100A9-treated PBMCs induced PG release from

18 bovine cartilage explants into culture supernatants. This effect was blocked by the

19 anti-TNF antibody D2E7, suggesting that the observed catabolic effects were

20 mediated primarily by endogenous TNF produced by activated PBMCs. Consistent

21 with these in vitro findings, treatment of the hTNF-Tg mice, Tg197, with D2E7

22 resulted in protection from TNF-induced depletion of PG from the articular cartilage.

23 Collectively our data provide a mechanistic explanation for the role of TNF in

24 cartilage damage in RA. We demonstrated that activated PBMCs are a major source

25 of TNF, whereas stromal cells are directly activated by TNF and express large

TNF directly contributes to cartilage damage in arthritis 
1 amounts of MMPs and IL-6. MMP production by chondrocytes and synovial

2 fibroblasts triggers PG depletion from cartilage. Our findings are in agreement with

3 the results of an elegant bone marrow transplant study in hTNF-Tg mice that

4 demonstrated the essential role of TNF-dependent activation of the mesenchymal but

5 not the haematopoietic compartment in the development of arthritis (54). Stromal

6 cells produced significantly higher levels of proteases and IL-6 than blood cells, and

7 expression persisted for days. Ineffective termination of inflammatory signalling in

$8 \quad$ RA synovial fibroblasts was demonstrated previously and can be explained by

9 increased chromatin accessibility in the promoters of inflammatory genes (55). We

10 found that, similar to FLS, chondrocytes exhibited sustained responses to TNF. One

11 possible explanation is that, unlike in immune cells or epithelium where homeostatic

12 mechanisms effectively control responses to inflammatory factors, stromal cells that

13 are normally not exposed to the environment mount an exaggerated or continuous

14 response, leading to propagation of joint inflammation and damage in RA.

\section{Conclusions}

16 We demonstrate that TNF directly triggers a catabolic program in human chondrocytes

17 leading to cartilage damage. Our data also show distinct roles for the major cell 18 populations present in the inflamed joints in the triggering and propagation of cartilage

19 damage and suggest that neutralization of TNF produced by PBMCs infiltrating 20 inflamed joints decreases catabolic activity of chondrocytes and fibroblasts, which 21 contributes to the cartilage protective effects of anti-TNF biologics in RA. 


\section{Abbreviations}

2 TNF: tumor necrosis factor; RA: rheumatoid arthritis; PG: proteoglycan; FLS:

3 fibroblast-like synoviocytes; PBMC: peripheral blood mononuclear cells; MMP:

4 metalloproteinase; IgG: Immunoglobulin G; FBS: fetal bovine serum; MSD:

5 mesoscale discovery; OD: optical density; hTNF-Tg mice: human TNF transgenic

6 mice; IL: interleukin; LPS: lipopolysaccharide; DAMP: damage-associated molecular

7 patterns; OCT compound: optimal cut temperature compound; ECM: extra-cellular

8 matrix; DMARD: disease-modifying antirheumatic drug

\section{Declarations:}

11 Ethics approval and consent to participate

12 Experimental protocols were accepted by the Institutional Committee for Animal Care

13 and Use and Experimental Code: S1.53 was given by the Veterinarian of Hellenic

14 Pasteur Institute, fully approved status animal program by Abbott's Corporate Animal

15 Welfare Committee (July 25, 2012). Annual license for this study: 2369/29-03-2012

16 issued by the Veterinary Department of the Athens Prefecture.

17 Consent for publication

18 Not applicable

19 Availability of data and materials

20 The datasets used and/or analysed during the current study are available from the

21 corresponding author on reasonable request.

\section{Competing interests}

23 All authors are employees of AbbVie and authors may own AbbVie stock. The

24 design, study conduct, and financial support for this research were provided by

TNF directly contributes to cartilage damage in arthritis 
1 AbbVie. AbbVie participated in the interpretation of data, review, and approval of the

2 publication.

\section{Funding}

4 This work was supported by funding from AbbVie, Inc. The funder provided support

5 in the form of salaries for all authors and the cost of animal study at Hellenic Pasteur

6 Institute.

\section{Author's' contributions}

8 AY conceived, designed and performed the study and wrote the manuscript. TM

9 carried out IHC and image analysis for the in vitro cartilage destruction assay. ZK

10 conceived and oversaw the project and contributed to the writing of manuscript. All

11 authors reviewed and approved the final manuscript.

\section{Acknowledgements}

13 We thank S. Haralambous and personal from the Laboratory of Transgenic

14 Technology, Hellenic Pasteur Institute for help with in vivo studies; C. Nelson for the

15 technical assistance with sample processing; A. Goodearl, M. Levesque, J. Seagal and

16 D. Hu for critical review of the manuscript.

\section{References}

1. Firestein GS, McInnes IB. Immunopathogenesis of Rheumatoid Arthritis. Immunity. 2017;46(2):183-96.

2. Angelotti F, Parma A, Cafaro G, Capecchi R, Alunno A, Puxeddu I. One year in review 2017: pathogenesis of rheumatoid arthritis. Clin Exp Rheumatol. 2017;35(3):368-78.

3. Smolen JS, Aletaha D, Barton A, Burmester GR, Emery P, Firestein GS, et al. Rheumatoid arthritis. Nat Rev Dis Primers. 2018;4:18001.

4. Schett G, Elewaut D, McInnes IB, Dayer JM, Neurath MF. How cytokine networks fuel inflammation: Toward a cytokine-based disease taxonomy. Nat Med. 2013;19(7):822-4.

5. Pap T, Korb-Pap A. Cartilage damage in osteoarthritis and rheumatoid arthritis--two unequal siblings. Nat Rev Rheumatol. 2015;11(10):606-15.

6. Polzer K, Schett G, Zwerina J. The lonely death: chondrocyte apoptosis in TNF-induced arthritis. Autoimmunity. 2007;40(4):333-6.

TNF directly contributes to cartilage damage in arthritis 
17 Goldring SR. Pathogenesis of bone and cartilage destruction in rheumatoid

2 arthritis. Rheumatology (Oxford). 2003;42 Suppl 2:ii11-6.

3 8. Klimiuk PA, Sierakowski S, Domyslawska I, Chwiecko J. Effect of repeated

4 infliximab therapy on serum matrix metalloproteinases and tissue inhibitors of

5 metalloproteinases in patients with rheumatoid arthritis. J Rheumatol.

6 2004;31(2):238-42.

7 9. Cho TJ, Lehmann W, Edgar C, Sadeghi C, Hou A, Einhorn TA, et al. Tumor

8 necrosis factor alpha activation of the apoptotic cascade in murine articular

9 chondrocytes is associated with the induction of metalloproteinases and specific pro-

10 resorptive factors. Arthritis Rheum. 2003;48(10):2845-54.

11 10. Firestein GS. Evolving concepts of rheumatoid arthritis. Nature.

12 2003;423(6937):356-61.

13 11. Bottini N, Firestein GS. Duality of fibroblast-like synoviocytes in RA: passive 14 responders and imprinted aggressors. Nat Rev Rheumatol. 2013;9(1):24-33.

15 12. Korb-Pap A, Stratis A, Muhlenberg K, Niederreiter B, Hayer S, Echtermeyer

$16 \mathrm{~F}$, et al. Early structural changes in cartilage and bone are required for the attachment

17 and invasion of inflamed synovial tissue during destructive inflammatory arthritis.

18 Ann Rheum Dis. 2012;71(6):1004-11.

19 13. Peters MA, Wendholt D, Strietholt S, Frank S, Pundt N, Korb-Pap A, et al.

20 The loss of alpha2beta1 integrin suppresses joint inflammation and cartilage

21 destruction in mouse models of rheumatoid arthritis. Arthritis Rheum.

22 2012;64(5):1359-68.

23 14. Lefevre S, Knedla A, Tennie C, Kampmann A, Wunrau C, Dinser R, et al. Synovial fibroblasts spread rheumatoid arthritis to unaffected joints. Nat Med. 2009;15(12):1414-20.

15. Pap T, Aupperle KR, Gay S, Firestein GS, Gay RE. Invasiveness of synovial fibroblasts is regulated by p53 in the SCID mouse in vivo model of cartilage invasion. Arthritis Rheum. 2001;44(3):676-81.

16. Pretzel D, Pohlers D, Weinert S, Kinne RW. In vitro model for the analysis of synovial fibroblast-mediated degradation of intact cartilage. Arthritis Res Ther. 2009;11(1):R25.

17. Zwerina J, Redlich K, Polzer K, Joosten L, Kronke G, Distler J, et al. TNFinduced structural joint damage is mediated by IL-1. Proc Natl Acad Sci U S A. 2007;104(28):11742-7.

18. Schuerwegh AJ, Dombrecht EJ, Stevens WJ, Van Offel JF, Bridts CH, De Clerck LS. Influence of pro-inflammatory (IL-1 alpha, IL-6, TNF-alpha, IFN-gamma) and anti-inflammatory (IL-4) cytokines on chondrocyte function. Osteoarthritis Cartilage. 2003;11(9):681-7.

19. Manicourt DH, Poilvache P, Van Egeren A, Devogelaer JP, Lenz ME, Thonar EJ. Synovial fluid levels of tumor necrosis factor alpha and oncostatin M correlate with levels of markers of the degradation of crosslinked collagen and cartilage aggrecan in rheumatoid arthritis but not in osteoarthritis. Arthritis Rheum. 2000;43(2):281-8.

20. Breedveld FC, Weisman MH, Kavanaugh AF, Cohen SB, Pavelka K, van Vollenhoven R, et al. The PREMIER study: A multicenter, randomized, double-blind clinical trial of combination therapy with adalimumab plus methotrexate versus methotrexate alone or adalimumab alone in patients with early, aggressive rheumatoid arthritis who had not had previous methotrexate treatment. Arthritis Rheum. 2006;54(1):26-37. 
1 21. Smolen JS, van der Heijde DM, Keystone EC, van Vollenhoven RF, Goldring

$2 \mathrm{MB}$, Guerette B, et al. Association of joint space narrowing with impairment of

3 physical function and work ability in patients with early rheumatoid arthritis:

4 protection beyond disease control by adalimumab plus methotrexate. Ann Rheum Dis.

5 2013;72(7):1156-62.

6 22. Dohn UM, Ejbjerg B, Boonen A, Hetland ML, Hansen MS, Knudsen LS, et al.

7 No overall progression and occasional repair of erosions despite persistent

8 inflammation in adalimumab-treated rheumatoid arthritis patients: results from a

9 longitudinal comparative MRI, ultrasonography, CT and radiography study. Ann

10 Rheum Dis. 2011;70(2):252-8.

11 23. Chatzidionysiou K, Emamikia S, Nam J, Ramiro S, Smolen J, van der Heijde

12 D, et al. Efficacy of glucocorticoids, conventional and targeted synthetic disease-

13 modifying antirheumatic drugs: a systematic literature review informing the 2016

14 update of The EULAR recommendations for the management of rheumatoid arthritis.

15 Annals of the Rheumatic Diseases. 2017;76(6):1102-7.

16 24. Hayer S, Bauer G, Willburger M, Sinn K, Alasti F, Plasenzotti R, et al.

17 Cartilage damage and bone erosion are more prominent determinants of functional

18 impairment in longstanding experimental arthritis than synovial inflammation. Dis

19 Model Mech. 2016;9(11):1329-38.

20 25. Aletaha D, Smolen J, Ward MM. Measuring function in rheumatoid arthritis:

21 Identifying reversible and irreversible components. Arthritis Rheum.

22 2006;54(9):2784-92.

23 26. Niki Y, Takeuchi T, Nakayama M, Nagasawa H, Kurasawa T, Yamada H, et

24 al. Clinical significance of cartilage biomarkers for monitoring structural joint damage

in rheumatoid arthritis patients treated with anti-TNF therapy. PLoS One. 2012;7(5):e37447.

27. Brown AK, Quinn MA, Karim Z, Conaghan PG, Peterfy CG, Hensor E, et al. Presence of significant synovitis in rheumatoid arthritis patients with diseasemodifying antirheumatic drug-induced clinical remission: evidence from an imaging study may explain structural progression. Arthritis Rheum. 2006;54(12):3761-73. 28. Ferrari-Lacraz S, Ferrari S. Do RANKL inhibitors (denosumab) affect inflammation and immunity? Osteoporos Int. 2011;22(2):435-46.

29. Smolen JS, Landewe R, Bijlsma J, Burmester G, Chatzidionysiou K, Dougados M, et al. EULAR recommendations for the management of rheumatoid arthritis with synthetic and biological disease-modifying antirheumatic drugs: 2016 update. Ann Rheum Dis. 2017;76(6):960-77.

30. Aletaha D, Alasti F, Smolen JS. Optimisation of a treat-to-target approach in rheumatoid arthritis: strategies for the 3-month time point. Ann Rheum Dis. 2016;75(8):1479-85.

31. Smolen JS, Aletaha D, Bijlsma JW, Breedveld FC, Boumpas D, Burmester G, et al. Treating rheumatoid arthritis to target: recommendations of an international task force. Ann Rheum Dis. 2010;69(4):631-7.

32. Smolen JS, Aletaha D. Rheumatoid arthritis therapy reappraisal: strategies, opportunities and challenges. Nat Rev Rheumatol. 2015;11(5):276-89.

33. Keffer J, Probert L, Cazlaris H, Georgopoulos S, Kaslaris E, Kioussis D, et al. Transgenic mice expressing human tumour necrosis factor: a predictive genetic model of arthritis. EMBO J. 1991;10(13):4025-31.

34. Karsdal MA, Woodworth T, Henriksen K, Maksymowych WP, Genant H, Vergnaud $\mathrm{P}$, et al. Biochemical markers of ongoing joint damage in rheumatoid 
1 arthritis--current and future applications, limitations and opportunities. Arthritis Res 2 Ther. 2011;13(2):215.

3 35. Otero M, Goldring MB. Cells of the synovium in rheumatoid arthritis.

4 Chondrocytes. Arthritis Res Ther. 2007;9(5):220.

5 36. Muller-Ladner U, Kriegsmann J, Franklin BN, Matsumoto S, Geiler T, Gay

6 RE, et al. Synovial fibroblasts of patients with rheumatoid arthritis attach to and

7 invade normal human cartilage when engrafted into SCID mice. Am J Pathol.

8 1996;149(5):1607-15.

9 37. Bartok B, Firestein GS. Fibroblast-like synoviocytes: key effector cells in 10 rheumatoid arthritis. Immunol Rev. 2010;233(1):233-55.

11 38. Nevius E, Gomes AC, Pereira JP. Inflammatory Cell Migration in Rheumatoid 12 Arthritis: A Comprehensive Review. Clin Rev Allergy Immunol. 2016;51(1):59-78.

13 39. Yang, Han Z, Oppenheim JJ. Alarmins and immunity. Immunol Rev.

$14 \quad 2017 ; 280(1): 41-56$.

15 40. Nefla M, Holzinger D, Berenbaum F, Jacques C. The danger from within:

16 alarmins in arthritis. Nat Rev Rheumatol. 2016;12(11):669-83.

17 41. Joosten LA, Helsen MM, van de Loo FA, van den Berg WB. Anticytokine

18 treatment of established type II collagen-induced arthritis in DBA/1 mice. A

19 comparative study using anti-TNF alpha, anti-IL-1 alpha/beta, and IL-1Ra. Arthritis

20 Rheum. 1996;39(5):797-809.

21 42. Cohen SB, Moreland LW, Cush JJ, Greenwald MW, Block S, Shergy WJ, et

22 al. A multicentre, double blind, randomised, placebo controlled trial of anakinra

(Kineret), a recombinant interleukin 1 receptor antagonist, in patients with rheumatoid arthritis treated with background methotrexate. Ann Rheum Dis. 2004;63(9):1062-8. 43. Schett G, Dayer JM, Manger B. Interleukin-1 function and role in rheumatic disease. Nat Rev Rheumatol. 2016;12(1):14-24.

44. Abramson SB, Amin A. Blocking the effects of IL-1 in rheumatoid arthritis protects bone and cartilage. Rheumatology (Oxford). 2002;41(9):972-80.

45. Jiang Y, Genant HK, Watt I, Cobby M, Bresnihan B, Aitchison R, et al. A multicenter, double-blind, dose-ranging, randomized, placebo-controlled study of recombinant human interleukin-1 receptor antagonist in patients with rheumatoid arthritis: radiologic progression and correlation of Genant and Larsen scores. Arthritis Rheum. 2000;43(5):1001-9.

46. Humby F, Kelly S, Hands R, Rocher V, DiCicco M, Ng N, et al. Use of ultrasound-guided small joint biopsy to evaluate the histopathologic response to rheumatoid arthritis therapy: recommendations for application to clinical trials. Arthritis Rheumatol. 2015;67(10):2601-10.

47. Croft A, Naylor A, Filer A, Buckley C. Effect of cartilage implantation on synovial fibroblasts from patients with rheumatoid arthritis. Lancet. 2014;383:38-. 48. Mizoguchi F, Slowikowski K, Wei K, Marshall JL, Rao DA, Chang SK, et al. Functionally distinct disease-associated fibroblast subsets in rheumatoid arthritis. Nat Commun. 2018;9(1):789.

49. Hammer HB, Odegard S, Syversen SW, Landewe R, van der Heijde D, Uhlig $\mathrm{T}$, et al. Calprotectin (a major S100 leucocyte protein) predicts 10-year radiographic progression in patients with rheumatoid arthritis. Ann Rheum Dis. 2010;69(1):150-4. 50. Odink K, Cerletti N, Bruggen J, Clerc RG, Tarcsay L, Zwadlo G, et al. Two calcium-binding proteins in infiltrate macrophages of rheumatoid arthritis. Nature. 1987;330(6143):80-2. 
1 51. van Lent PL, Grevers L, Blom AB, Sloetjes A, Mort JS, Vogl T, et al.

2 Myeloid-related proteins S100A8/S100A9 regulate joint inflammation and cartilage

3 destruction during antigen-induced arthritis. Ann Rheum Dis. 2008;67(12):1750-8.

4 52. Donato R, Cannon BR, Sorci G, Riuzzi F, Hsu K, Weber DJ, et al. Functions

5 of S100 proteins. Curr Mol Med. 2013;13(1):24-57.

6 53. van Lent PL, Grevers LC, Schelbergen R, Blom A, Geurts J, Sloetjes A, et al.

7 S100A8 causes a shift toward expression of activatory Fcgamma receptors on

8 macrophages via toll-like receptor 4 and regulates Fcgamma receptor expression in

9 synovium during chronic experimental arthritis. Arthritis Rheum. 2010;62(11):3353-

1064.

11 54. Bluml S, Binder NB, Niederreiter B, Polzer K, Hayer S, Tauber S, et al.

12 Antiinflammatory effects of tumor necrosis factor on hematopoietic cells in a murine 13 model of erosive arthritis. Arthritis Rheum. 2010;62(6):1608-19.

14 55. Lee A, Qiao Y, Grigoriev G, Chen J, Park-Min KH, Park SH, et al. Tumor 15 necrosis factor alpha induces sustained signaling and a prolonged and unremitting 16 inflammatory response in rheumatoid arthritis synovial fibroblasts. Arthritis Rheum. $17 \quad 2013 ; 65(4): 928-38$.

\section{Figure Legends}

Figure 1. TNF upregulates IL-6 and MMP in human chondrocytes and increases PG depletion from bovine cartilage

(A) Levels of IL-6, MMP1 and MMP3 proteins produced by human cartilage explants (upper panel, $n=3$ ) or primary chondrocytes (lower panel, $n=7$ ) isolated from healthy individuals stimulated with recombinant human TNF $(10 \mathrm{ng} / \mathrm{ml})$ for the indicated times were measured by MSD assay. Data are shown as mean \pm SEM. Significant differences from untreated control collected at the same time point are depicted: ${ }^{*}, p<0.05 ;{ }^{* *}, p<0.01 ;{ }^{* * * *}, p<0.0001$ (two-way ANOVA with Sidak's post-test for multiple comparisons). (B) PG content in bovine cartilage explants (four replicates/condition) cultured with or without $10 \mathrm{ng} / \mathrm{ml}$ of recombinant human TNF or IL-1 $\beta$ (positive control) for 14 days was evaluated using toluidine blue staining. The upper panel shows representative histological samples where PG in control untreated samples stained dark blue, and loss of blue colour represents PG depletion from the cartilage. The lower panel depicts results of quantitative image analysis of the stained cartilage sections presented as a percent of untreated cartilage (100\%, dotted line). Bars show the mean \pm SEM of five independent experiments. ${ }^{*}=p<0.05$ as compared to untreated control (one-way ANOVA with Dunn's multiple comparison test).

(C) Effects of IL-1 $\beta$ and TNF on IL- 6 and MMPs production by human isolated chondrocytes described in $(A)$ at 48 hours of culture. Data are shown as mean \pm SEM, significant differences between two cytokines are shown: ${ }^{*}=p<0.05$ (two-way ANOVA with Sidak's post-test for multiple comparisons).

\section{Figure 2. Effects of co-culture with FLS and TNF blockade on TNF-mediated cartilage destruction}

(A) Quantitative analysis of PG content in bovine cartilage explant monocultures $(n=5)$ or cocultures with FLS isolated from synovium of RA patients $(n=4)$ or individuals without joint disease $(n=5)$. Cultures were exposed to $10 \mathrm{ng} / \mathrm{ml}$ of TNF for 14 days or left untreated, explants were processed and stained with toluidine blue as in Fig. 1B. Results are presented as a percent of untreated cartilage (100\%, dotted line). (B) IL-6 and MMPs production by RA

TNF directly contributes to cartilage damage in arthritis 
and non-diseased FLS in supernatants collected from bovine cartilage explant co-cultures as in (A) measured by MSD assay. For both (A) and (B) data are shown as mean \pm SEM, significant differences from untreated controls are shown: ${ }^{*}=p<0.05 ;{ }^{* *}=p<0.01 ; * * *$, $\mathrm{p}<0.001 ;{ }^{* * *}, \mathrm{p}<0.0001$ (repeated measure two-way ANOVA with Sidak's post-test for multiple comparisons). (C) Effect of TNF blockade on PG depletion from the cartilage were tested on the same co-cultures of bovine cartilage explants with human FLS as in (A). $\mathrm{FLS} /$ cartilage co-cultures were exposed to $10 \mathrm{ng} / \mathrm{ml}$ of TNF or IL-1 $\beta$ for 3 days and thereafter, $5 \mu \mathrm{g} / \mathrm{ml}$ of D2E7 or IgG1 (negative control) were added to some of the wells. Explants were processed and analysed as described for panel (A). Results are presented as a percent of untreated cartilage (100\%, dotted line). (D) Effect of TNF neutralization by D2E7 on IL-6 and MMPs production by FLS in the same co-culture experiments as in (C). For both (C) and (D) data are shown as mean \pm SEM, significant differences from $\lg G 1$ treated cultures are depicted: ${ }^{*}=p<0.05 ;{ }^{* *}=p<0.01 ; * * * *, p<0.0001$ (repeated measure two-way ANOVA with Sidak's post-test for multiple comparisons).

\section{Figure 3. Characterization of TNF production and TNF responses in chondrocytes, FLS and PBMCs}

All experiments described in this figure were performed with primary human cells isolated from individuals without history of joint diseases. (A) TNF production by human $\operatorname{FLS}(n=4)$ and chondrocytes $(n=3)$ cultured in the presence or absence of $10 \mathrm{ng} / \mathrm{ml}$ of recombinant human IL-1 $\beta$ or LPS for $48 \mathrm{~h}$. (B) Comparison of TNF levels produced by human FLS $(n=5)$, chondrocytes $(n=9)$ or PBMCs $(n=5)$ in response to $48 \mathrm{~h}$ treatment with $10 \mu \mathrm{g} / \mathrm{ml}$ of alarmins: S100A8 and S1009, $10 \mathrm{ng} / \mathrm{ml}$ of IL-1 $\beta$ or LPS (positive control). (C) IL-6 and MMP1 production by human FLS $(n=6)$, chondrocytes $(n=7)$ or PBMCs $(n=5)$ in response to stimulation with $10 \mathrm{ng} / \mathrm{ml}$ of TNF. All data are shown as mean \pm SEM, significant difference from untreated control is depicted: ${ }^{*}=p<0.05 ;^{* *}=p<0.01$ (Kruskal-Wallis test with Dunn's multiple comparison post-test).

\section{Figure 4. TNF neutralization protects cartilage in vitro and in vivo, in hTNF-Tg mouse model of arthritis}

(A) Bovine cartilage explants were co-cultured with PBMCs isolated from the whole blood of healthy individuals with or without $10 \mu \mathrm{g} / \mathrm{ml}$ of alarmins: S100A8, S1009 or TNF as a positive control. D2E7 or isotype control (hlgG1) were added at $5 \mu \mathrm{g} / \mathrm{ml}$ to some wells. After 7 days of culture, supernatants were collected, and levels of PG depleted from the cartilage into the culture media were measured. Results are presented as a fold over PG levels in untreated samples. Data are shown as mean \pm SEM of 3 independent experiments. Dotted line represents untreated control. (B) Human PBMCs alone (left panel, $n=4$ ) or in co-culture with bovine cartilage (right panel, $\mathrm{n}=3$ ) were stimulated with $10 \mu \mathrm{g} / \mathrm{ml}$ of alarmins: S100A8 and S1009 or left untreated in the presence of $5 \mu \mathrm{g} / \mathrm{ml}$ of D2E7 or isotype control (hlgG1), supernatants were collected and levels of TNF were measured by MSD assay. Data are shown as mean \pm SEM, significant difference between D2E7 and isotype control is shown: ${ }^{*}=p<0.05$ (two-way ANOVA with Tukey's multiple comparison test). (C) Representative alcian blue stained sections from ankle joints of hTNF-Tg mice treated for 12 weeks with $1 \mathrm{mg} / \mathrm{kg}$ once a week of anti-TNF $\alpha$ antibody D2E7 ( $n=12)$ or placebo $(n=6)$ are shown on the upper panel (magnification 20x). Staining intensity of the articular cartilage (shown by asterisk) correlates with PG content. The lower panel depicts the results of quantitative image analysis of PG content (stained with alcian blue) in the articular cartilage of talus bones (area of analysis is outlined in red). Data for individual animals are shown as a percent of total cartilage area, significant difference between groups is shown: ${ }^{* *}=p<0.01$ (Mann-Whitney $U$ test).

TNF directly contributes to cartilage damage in arthritis 
Figures

A

Human cartilage
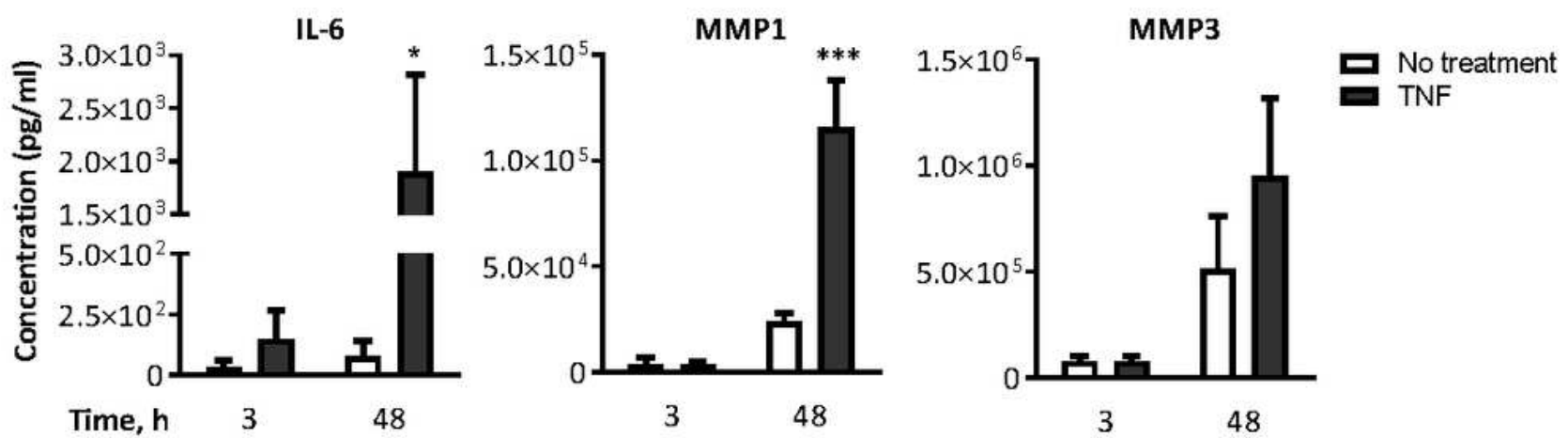

Human chondrocytes
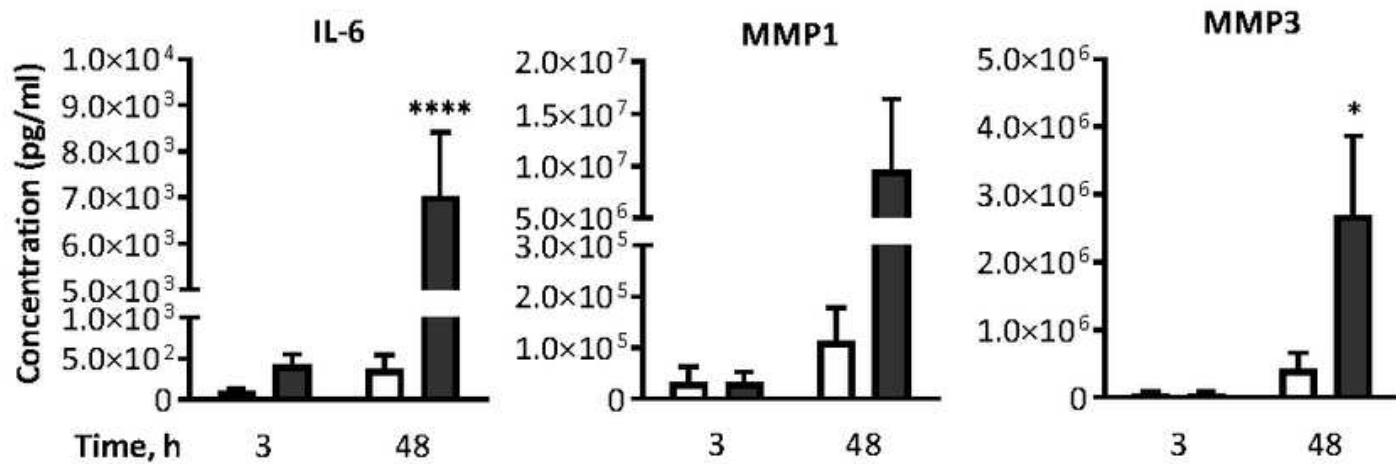

B

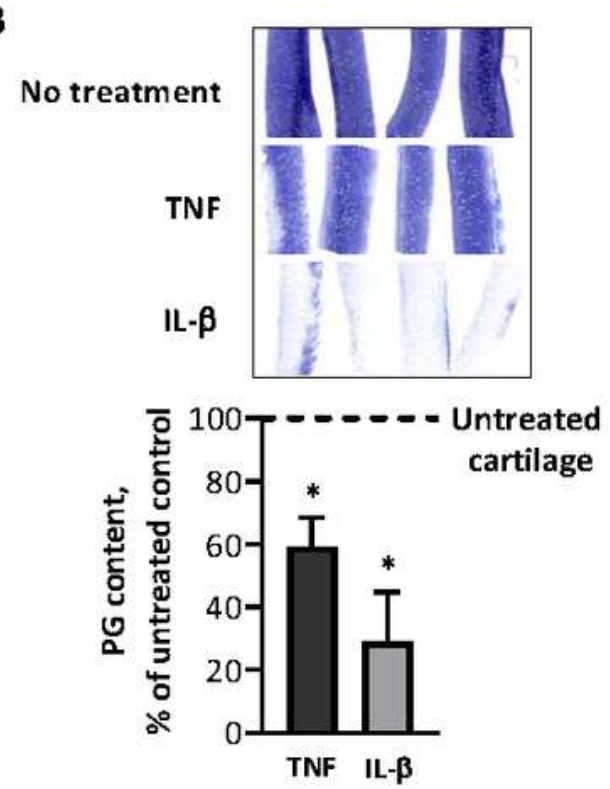

C

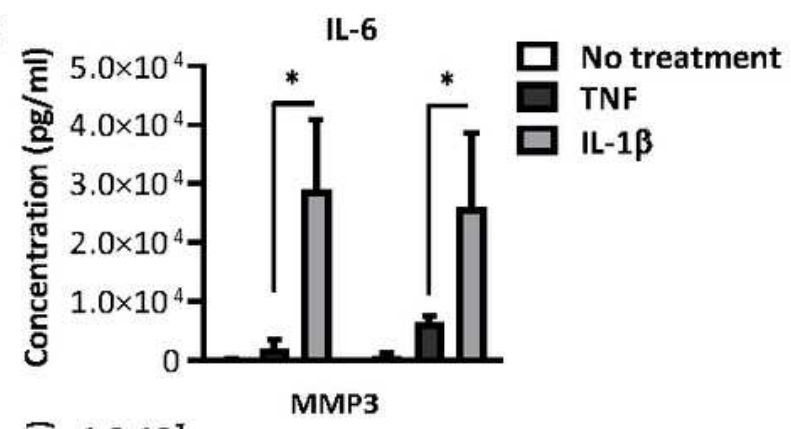

Figure 1

TNF upregulates IL-6 and MMP in human chondrocytes and increases PG depletion from bovine cartilage (A) Levels of IL-6, MMP1 and MMP3 proteins produced by human cartilage explants (upper panel, $n=3$ ) or primary chondrocytes (lower panel, $n=7$ ) isolated from healthy individuals stimulated with recombinant 
human TNF $(10 \mathrm{ng} / \mathrm{ml})$ for the indicated times were measured by MSD assay. Data are shown as mean \pm SEM. Significant differences from untreated control collected at the same time point are depicted: *, $\mathrm{p}<0.05 ; * \star, p<0.01 ; * \star \star \star, p<0.0001$ (two-way ANOVA with Sidak's post-test for multiple comparisons). (B) PG content in bovine cartilage explants (four replicates/condition) cultured with or without $10 \mathrm{ng} / \mathrm{ml}$ of recombinant human TNF or IL-1 $\beta$ (positive control) for 14 days was evaluated using toluidine blue staining. The upper panel shows representative histological samples where PG in control untreated samples stained dark blue, and loss of blue colour represents PG depletion from the cartilage. The lower panel depicts results of quantitative image analysis of the stained cartilage sections presented as a percent of untreated cartilage $(100 \%$, dotted line). Bars show the mean \pm SEM of five independent experiments. ${ }^{*}=p<0.05$ as compared to untreated control (one-way ANOVA with Dunn's multiple comparison test). (C) Effects of IL-1 $\beta$ and TNF on IL- 6 and MMPs production by human isolated chondrocytes described in (A) at 48 hours of culture. Data are shown as mean \pm SEM, significant differences between two cytokines are shown: ${ }^{*}=p<0.05$ (two-way ANOVA with Sidak's post-test for multiple comparisons).

A

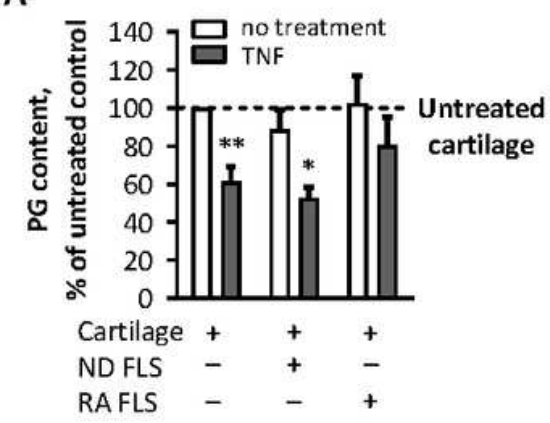

B

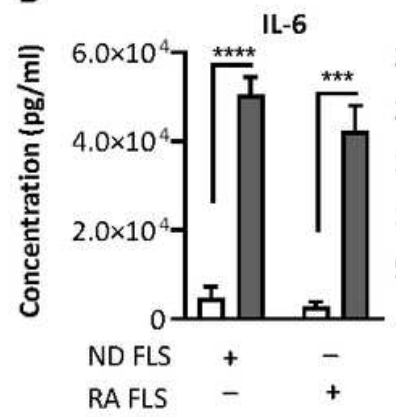

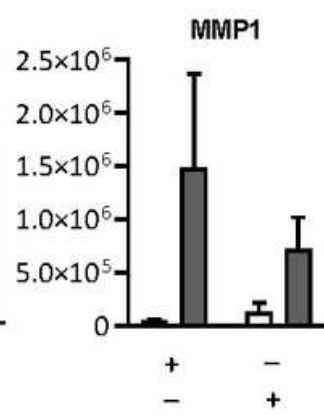

C Monoculture

Co-culture

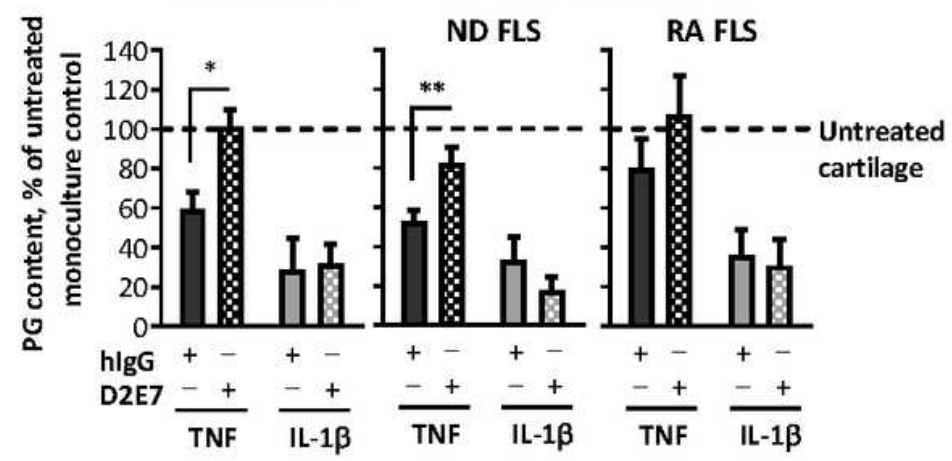

D

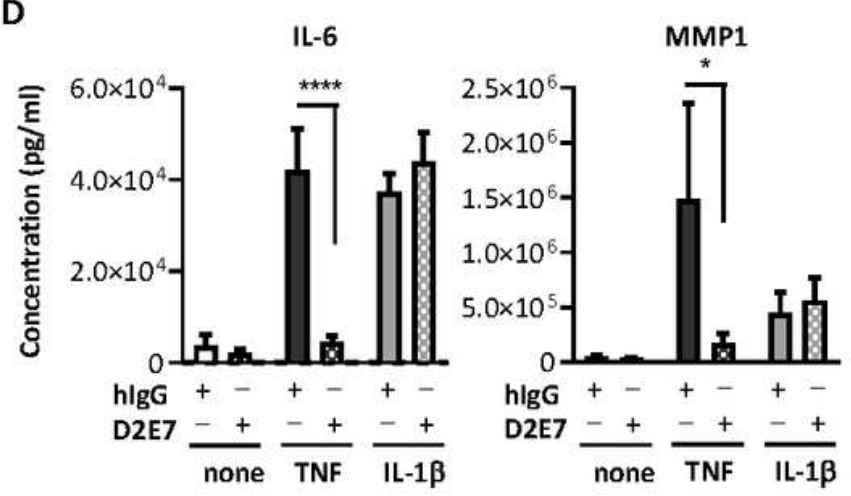

Figure 2

Effects of co-culture with FLS and TNF blockade on TNF-mediated cartilage destruction (A) Quantitative analysis of PG content in bovine cartilage explant monocultures $(n=5)$ or co-cultures with FLS isolated from synovium of RA patients $(n=4)$ or individuals without joint disease $(n=5)$. Cultures were exposed to $10 \mathrm{ng} / \mathrm{ml}$ of TNF for 14 days or left untreated, explants were processed and stained with toluidine blue as in Fig. 1B. Results are presented as a percent of untreated cartilage (100\%, dotted line). (B) IL-6 and MMPs production by RA and non-diseased FLS in supernatants collected from bovine cartilage explant 
co-cultures as in (A) measured by MSD assay. For both (A) and (B) data are shown as mean \pm SEM, significant differences from untreated controls are shown: * $=p<0.05 ; * \star=p<0.01 ; * \star \star, p<0.001 ; * \star \star *$, $p<0.0001$ (repeated measure two-way ANOVA with Sidak's post-test for multiple comparisons). (C) Effect of TNF blockade on PG depletion from the cartilage were tested on the same co-cultures of bovine cartilage explants with human FLS as in (A). FLS/cartilage co-cultures were exposed to $10 \mathrm{ng} / \mathrm{ml}$ of TNF or IL-1 $\beta$ for 3 days and thereafter, $5 \mu \mathrm{g} / \mathrm{ml}$ of D2E7 or IgG1 (negative control) were added to some of the wells. Explants were processed and analysed as described for panel (A). Results are presented as a percent of untreated cartilage (100\%, dotted line). (D) Effect of TNF neutralization by D2E7 on IL-6 and MMPs production by FLS in the same co-culture experiments as in (C). For both (C) and (D) data are shown as mean \pm SEM, significant differences from IgG1 treated cultures are depicted: ${ }^{*}=p<0.05 ; * \star=$ $p<0.01 ; * \star \star \star, p<0.0001$ (repeated measure two-way ANOVA with Sidak's post-test for multiple comparisons). 

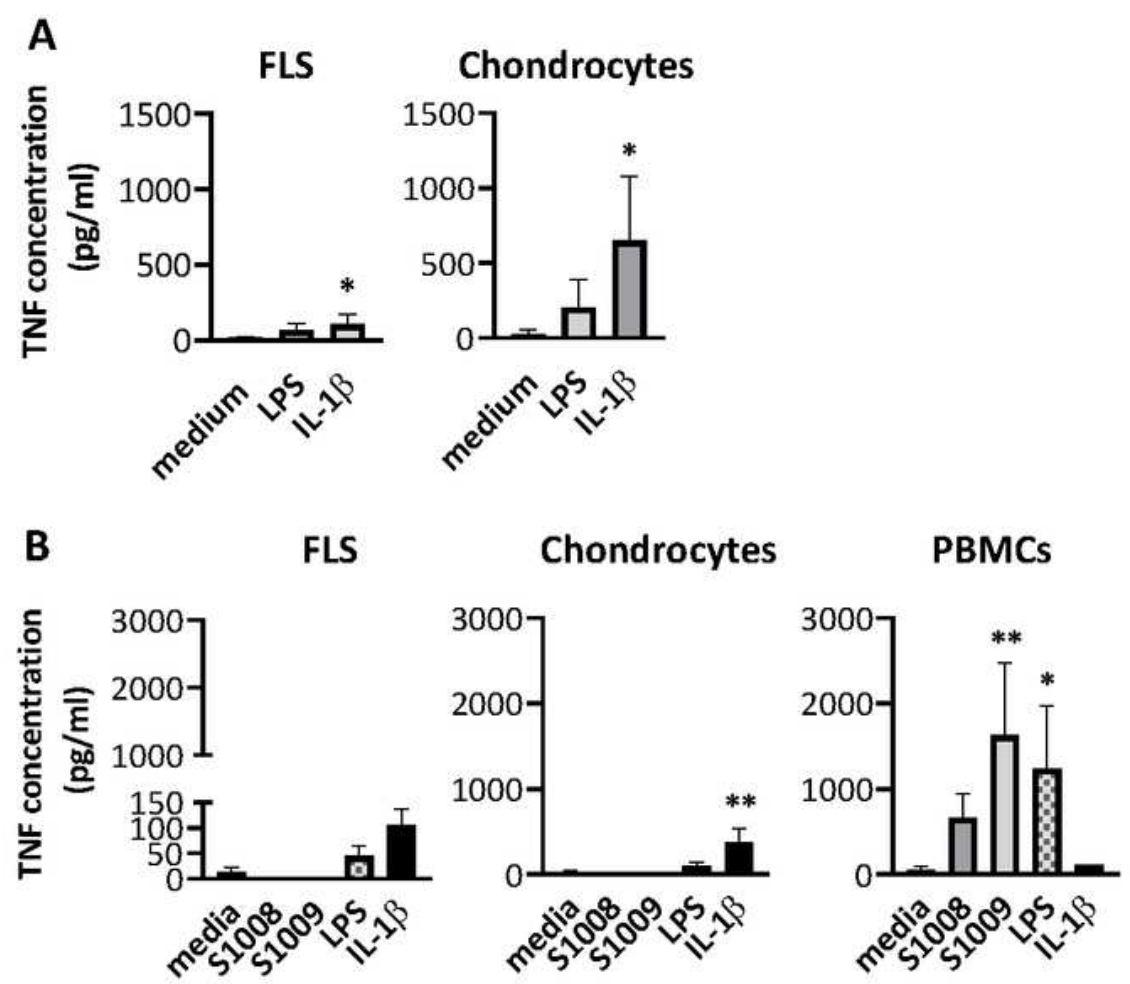

C

FLS

Chondrocytes

PBMCs

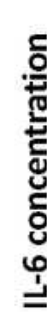
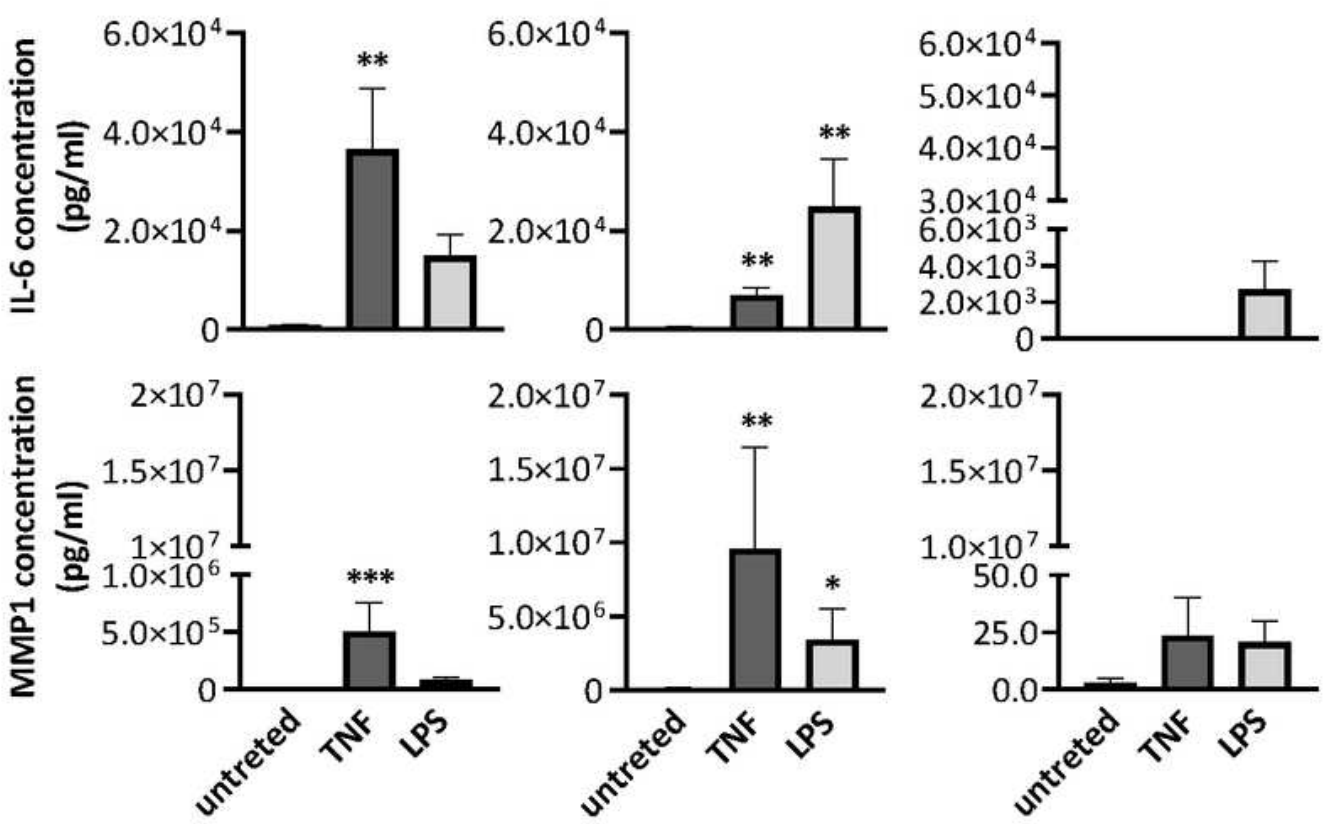

Figure 3

Characterization of TNF production and TNF responses in chondrocytes, FLS and PBMCs All experiments described in this figure were performed with primary human cells isolated from individuals without history of joint diseases. (A) TNF production by human FLS $(n=4)$ and chondrocytes $(n=3)$ cultured in the presence or absence of $10 \mathrm{ng} / \mathrm{ml}$ of recombinant human IL-1 $\beta$ or LPS for $48 \mathrm{~h}$. (B) Comparison of TNF levels produced by human FLS $(n=5)$, chondrocytes $(n=9)$ or PBMCs $(n=5)$ in response to $48 \mathrm{~h}$ treatment 
with $10 \mu \mathrm{g} / \mathrm{ml}$ of alarmins: S100A8 and S1009, $10 \mathrm{ng} / \mathrm{ml}$ of IL-1 $\beta$ or LPS (positive control). (C) IL-6 and MMP1 production by human FLS $(n=6)$, chondrocytes $(n=7)$ or PBMCs $(n=5)$ in response to stimulation with $10 \mathrm{ng} / \mathrm{ml}$ of TNF. All data are shown as mean \pm SEM, significant difference from untreated control is depicted: $*=p<0.05 ; * \star=p<0.01$ (Kruskal-Wallis test with Dunn's multiple comparison post-test).

A

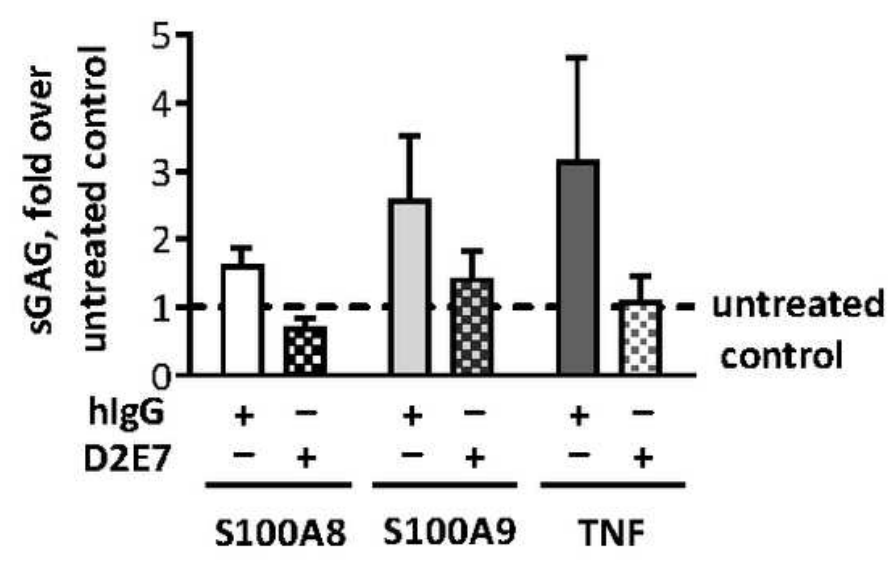

B

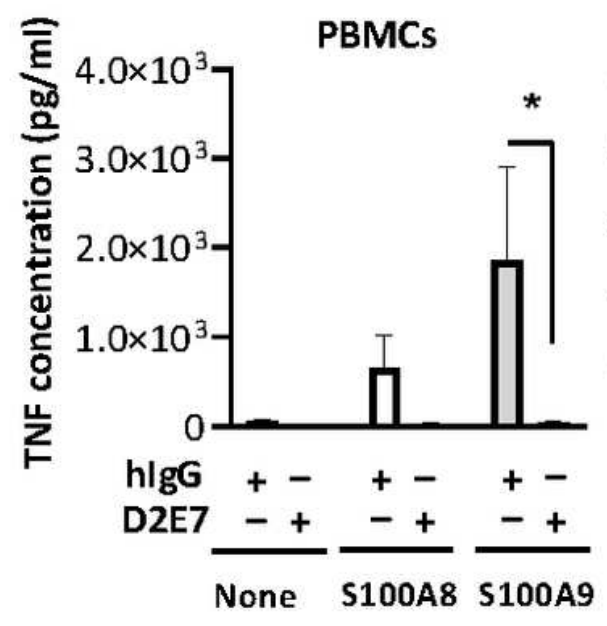

C

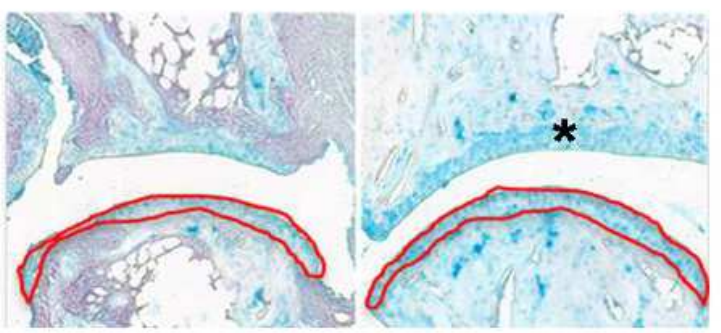

Placebo

D2E7

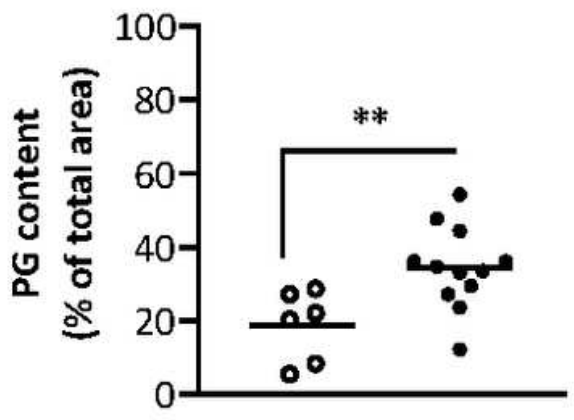

PL

D2E7

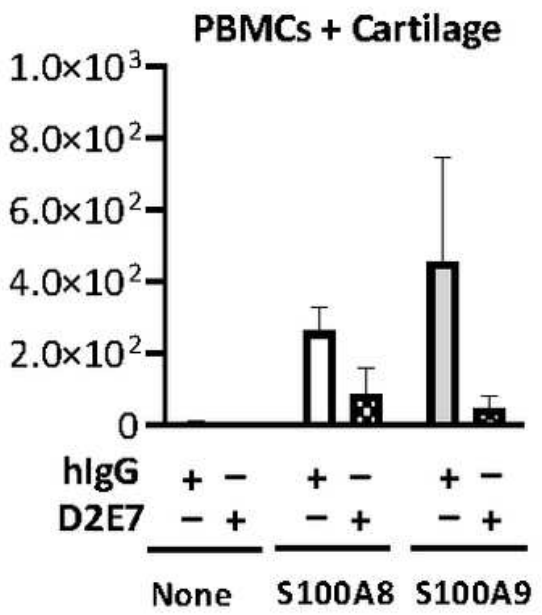

Figure 4

TNF neutralization protects cartilage in vitro and in vivo, in hTNF-Tg mouse model of arthritis (A) Bovine cartilage explants were co-cultured with PBMCs isolated from the whole blood of healthy individuals with or without $10 \mu \mathrm{g} / \mathrm{ml}$ of alarmins: S100A8, S1009 or TNF as a positive control. D2E7 or isotype control (hlgG1) were added at $5 \mu \mathrm{g} / \mathrm{ml}$ to some wells. After 7 days of culture, supernatants were collected, and levels of PG depleted from the cartilage into the culture media were measured. Results are presented as a fold over PG levels in untreated samples. Data are shown as mean \pm SEM of 3 independent experiments. Dotted line represents untreated control. (B) Human PBMCs alone (left panel, $n=4$ ) or in co-culture with 
bovine cartilage (right panel, $\mathrm{n}=3$ ) were stimulated with $10 \mu \mathrm{g} / \mathrm{ml}$ of alarmins: S100A8 and S1009 or left untreated in the presence of $5 \mu \mathrm{g} / \mathrm{ml}$ of D2E7 or isotype control (hlgG1), supernatants were collected and levels of TNF were measured by MSD assay. Data are shown as mean \pm SEM, significant difference between D2E7 and isotype control is shown: ${ }^{*}=p<0.05$ (two-way ANOVA with Tukey's multiple comparison test). (C) Representative alcian blue stained sections from ankle joints of hTNF-Tg mice treated for 12 weeks with $1 \mathrm{mg} / \mathrm{kg}$ once a week of anti-TNFa antibody D2E7 $(n=12)$ or placebo $(n=6)$ are shown on the upper panel (magnification 20x). Staining intensity of the articular cartilage (shown by asterisk) correlates with PG content. The lower panel depicts the results of quantitative image analysis of PG content (stained with alcian blue) in the articular cartilage of talus bones (area of analysis is outlined in red). Data for individual animals are shown as a percent of total cartilage area, significant difference between groups is shown: $* *=p<0.01$ (Mann-Whitney $U$ test). 